\title{
SUCCESSFUL ANESTHETIC MANAGEIMENT IN CESAREAN SECTION FOR PREGNANT WOIMEN WITH COVID 19
}

KEY WORDS:

\section{Salkthi Vignesh G*}

Senior Resident, Department Of Anaesthesia, Sri Lakshmi Narayana Institute Of Medical Sciences, Ossudu Agaram Village, Bharath Institute Of Higher Education And Research, Puducherry, India. *Corresponding Author

\section{INTRODUCTION}

In India the management of a COVID-19 is more challenging among pregnant women, as the virus is extremely contagious and can cause life threatening severe acute respiratory tract infection in 5\% patients and can spread to other mothers and health care personnel. ${ }^{1,2}$ There is increases risk of obstetric complication (Preterm labour, Premature rupture of membranes, Preeclampsia, caesarean section) among parturient covid 19 patients.3,4 It is noteworthy that the increase in the body temperature associated with COVID-19 (i.e., hyper-thermia) may lead to congenital anomalies if it occurs in the first trimester. ${ }^{5}$ This highlight the importance to establish covid 19 hospitals and operating rooms for parturient patients. In addition, there is no verified protocol for the anaesthetic aspect of providing care for pregnant women undergoing $\mathrm{C}$-section. As per literature review, central neuraxial blockade is the preferred technique to reduce the aerosol generation in such circumstances. ${ }^{1,2,6}$ In this article we highlight the anaesthetic aspect of C-section performed for a pregnant women diagnosed with covid 19.

\section{CASE REPORT}

A 27 years old COVID -positive women, G2Pl at 38 weeks of gestation, was referred to Sri Lakshmi Narayana Medical college after which she tested COVID-19 positive. At that time she had 2 day history of rhinorrhoea without fever, chills, dry cough or dyspnoea. Regarding her obstetrical history, her previous delivery was C-section in 2017 due to foetal distress. Her last menstrual period was on September 7,2019.

On admission, basic blood tests, electrocardiograms were done. Elevated erythrocytes sedimentation rate and Creactive protein were found, and the other investigations were unremarkable. During hospital stay, the obstetrics team was consulted and previous obstetric history was elicited. Her antenatal period was uneventful and shifted to an isolation room for safe confinement. Ultrasound revealed the oblique lie of the fetus and patient status, an elective lower segment caesarean section (LSCS) under subarachnoid block (SAB) was planned. The couple was counseled and they consented for the same. Antenatal records revealed no other co morbidities.

A separate maternity facility was created in the temporarily closed obstetric OPD to prevent exposure of other pregnant patients, with area for donning and doffing personal protective equipment (PPE) and a clear exit path including showering facility and an area for new-born resuscitation. The Minor Operation Room (OR) was fitted with an anaesthesia workstation supplied by type $\mathrm{E}$ and type $\mathrm{H}$ gas cylinders as there was no pipeline supply. In addition, we arranged an extra suction machine, anaesthetic, uterotonic and resuscitation drugs, spinal needles, facemasks, laryngoscopes including a video-laryngoscope with disposable blades, stylet, a transparent sheet to cover the patient, in case endotracheal intubation was required.

Level 3 PPE consisting of N95 respirators, impervious body suits with hoods, goggles, face visor, shoe covers and double layered medical gloves were worn by the entire team (obstetricians, anaesthesiologists, neonatologist, nursing staff and assistants) ${ }^{1,6-8}$ A nurse wearing PPE served as a communicator in the buffer area with a runner outside. The patient was then transferred to this facility with her face covered with a $\mathrm{N}-95$ respirator through a green corridor (a pre-planned route in which all other patient and personnel movement was stopped to minimize contamination).$^{6-8}$ Preanesthetic and airway examination was done after the patient arrived in the COVID area outside the OR. She was anxious (120/min pulse rate, $130 / 80 \mathrm{mmHg}$ blood pressure was, $96 \%$ room air oxygen saturation $\left(\mathrm{SpO}_{2}\right)$, had no dyspnea. She was reassured and explained about $\mathrm{SAB}$, the tachycardia settled. After attaching routine monitors (electrocardiogram, noninvasive blood pressure, $\mathrm{SpO}_{2}$ ), a 18G IV Cannula was secured, anti-aspiration prophylaxis given and patient preloaded with $500 \mathrm{ml}$ Ringer's lactate. The patient was put into the sitting position and painting was done. Local anesthesia was given ( $2 \mathrm{ml}$ of $2 \%$ lidocaine). Then SAB was administered in the L3-4 interspace with 25G Quincke needle. A block level up to T4 was achieved with $2.2 \mathrm{ml}$ hyperbaric bupivacaine $0.5 \%$ was administrated. The patient was kept in the sitting position for 30 s and then placed in supine position with her head raised. Her vitals were monitored and were stable without hypotension. Her $\mathrm{O}_{2}$ saturation was $98 \%$, her pulse was around 80, and her blood pressure was 124/68.

Subsequently, the obstetric team performed LSCS. The blood pressure was stable during the course of the procedure, and there was no need to give ephedrine. A healthy female baby was delivered and paediatrician performed the polymerase chain reaction COVID-19 swab and had 5 minutes Apgar score of 9 . The surgery lasted for 90 minutes and estimated blood loss was $600-800 \mathrm{ml}$. The mother was transferred to designated recovery room within the COVID area and looked after by the same residents and nursing staff. The baby was breast-fed. Once the Bromage score was 3 (about $135 \mathrm{~min}$ after the $S A B$ ), the patient was transferred back to her room. All team members exited the area after doffing under supervision.

After the procedure, the baby was handed over to the mother for nursing. She was instructed to keep on the N-95 respirator and maintain hand hygiene. The baby was tested on day 10 and 14 after birth using PCR and was negative both times. The mother was tested on day $14,21,22$ post-surgery and discharged home with the baby after 2 negative reports.

\section{DISCUSSION}

Pregnant women with positive COVID-19 tests are managed according to the severity of illness. Mild cases need continuous fetal heart rate monitoring as well as maintaining oxygen saturation $>95 \%$, while more severe cases are managed by ICU teams. Avoiding the use of betamethasone (i.e., glucocorticoids) is advised because it may be associated with increased risk for mortality and delayed viral clearance (as is the case with influenza virus). ${ }^{9}$

The clinical severity of COVID-19 and gestational age are the main factors in determining the appropriate time for delivery. Pregnant women with mild illness whose pregnancy is still preterm can wait until their infection status is negative. In mothers with severe illness, the risks and benefits should be 
weighed. If the pregnancy is past 32 weeks and delivery would help in improving a pregnant woman's respiratory condition, delivery is advised. However, if the gestation is premature or there is a risk of the respiratory condition worsening, then delivery can be delayed with constant fetal and maternal monitoring. ${ }^{9}$ The Centers for Disease Control and Prevention (CDC) recommends that all patients should inform hospitals in advance about their COVID-19 status and hospitals should screen for COVID-19 signs and symptoms in patients with contact history with confirmed or suspected cases. ${ }^{10}$ All health workers involved in the care of pregnant patients should wear surgical masks. Pregnant women with positive or suspected COVID-19 status should be cared for in negative pressure rooms before, during, and after delivery. ${ }^{9}$ Patients with COVID-19 can be delivered according to individual obstetric indications, and the active infection alone is not a reason to perform C-section ${ }^{9,11}$. Patients should be tested for COVID-19 if they are symptomatic, and an evaluation of the maternal and fetal status should be performed in order to balance the risks and benefits of delaying the delivery (i.e., CS or induction of labor) until the result of the test comes back. ${ }^{9}$

Use of neuraxial blockade techniques are advised during delivery of COVID-19 patients, whether by vaginal route or CS because these techniques will decrease the cardiopulmonary compromise caused by the stress of labor. Additionally, spinal anesthesia has advantages over general anesthesia for CS because it has lower rates of respiratory depression and it is not considered an aerosolizing procedure, so theoretically, it will decrease the need for personal protective equipment (i.e., decrease the chance of spread of the virus to health workers in the operating room).,12 Patients undergoing CS under spinal anesthesia should wear a regional mask during the procedure. Also, sedative drugs should be avoided due to their respiratory depressive effects, but if used, they should be given gradually until the desired effect is reached. If needed, supplemental oxygen should be given at the lowest flow possible. Although there is no evidence to suggest that COVID-19 might spread to the cerebrospinal fluid, contact with the patient's fluid should be avoided as much as possible while per-forming the spinal blockade. Epidural blood patch and sphenopalatine block are not advised to be used in case the patient develops a dural puncture headache. ${ }^{12}$ Postoperative patient controlled analgesia is best avoided to prevent respiratory depression. ${ }^{9}$

\section{CONCLUSION}

Special precautions should be considered when providing care for pregnant women undergoing CS. Spinal anesthesia is preferred over general anesthesia.

\section{REFERENCES}

1. Sorbello M,El-Boghdadly K,Di Giacinto I, Cataldo R, Esposito C, Falcetta S, et al. The Italian coronavirus disease 2019 outbreak: Recommendations from clinical practice. Anaesthesia 2020;75:724-32.

2. World Health Organization, Clinical management of severe acute respiratory infection (SARI) when COVID-19 disease is suspected. 2020. Available from: https://www.who.int/ publications-detail/clinical-management-ofsevere-acute-respiratory-infection-when-novel-coronavirus-(ncov)infection-is- suspected. [Last accessed on 2020 Apr 06].

3. Mullins E, Evans D, Viner RM et al: Coronavirus in pregnancy and delivery: Rapid review. Ultrasound Obstet Gynecol, 2020;55:586-82

4. Schwartz DA. An analysis of 38 pregnant women with COVID-19, their newborn infants, and maternal-fetal transmission of SARS-CoV-2: Maternal coronavirus infections and pregnancy outcomes. Arch Pathol Lab Med, 2020 [Online ahead of print]

5. Dukhovny S, Wilkins-Haug L: Open neural tube defects: Risk factors, prenatal screening and diagnosis, and pregnancy management. UpToDate. Waltham, MA:UpToDate;2018

6. Malhotra N, Bajwa SJ, Joshi M, Mehdiratta L, Trikha A. ISA advisory: COVID operation theatre- advisory and position statement of Indian society of anaesthesiologists (ISA National). Indian J Anaesth 2020;64:355-62.

7. Malhotra N, Joshi M, Datta R, Bajwa SJ, Mehdiratta L. Indian society of anaesthesiologists (ISA national) advisory and position statement regarding COVID-19. Indian J Anaesth 2020;64:259-63.

8. Chen R, Zhang Y, Huang L, Cheng BH, Xia ZY, Meng QT. Safety and efficacy of different anaesthetic regimens for parturients with COVID-19 undergoing Caesrean delivery: $A$ case series of 17 patients. Can J Anaesth 2020;67:65563.

9. Rothe C, Schunk M, Sothmann P et al: Transmission of 2019-nCoV infec- tion from an asymptomatic contact in Germany.N Engl J Med, 2020;3 82:970-71

10. Center for Disease Control and Prevention [Internet]. National Center for Immunization and Respiratory Diseases (NCIRD), Division of Viral Diseases. Considerations for Inpatient Obstetric Healthcare Settings. https://www. cdc.gov/coronavirus/2019-ncov/hcp/inpatient-obstetric-healthcare-guidance.html

11. World Health Organization [Internet]. Clinical management of severe acute respiratory infection when COVID-19 is suspected: Interim Guidance. https:// www who.int/publications-detail/clinical-manag ement-of-severeacute-respi- ratory-infection-when-novel-coronavirus-(ncov)-infection-issuspected

12. Society for Obstetric Anesthesia and Perinatology [Internet]. Interim Considerations for Obstetric Anesthesia Care Related to COVID-19. https://soap.org/education/provider-education/expert-summaries/ interim-considerations-for-obstetric-anesthesia-care-related-to-covidl 9 